\title{
Dental Laser in The Covid-19 Era
}

\author{
Natalia Elson, Irene Brandes, and Steven Resnick
}

\section{ABSTRACT}

Despite the many historical events during the year 2020, this period of time will forever be remembered as the year of COVID-19. Since its onset in November 2019, The Sars-CoV-2 has made an enormous impact across all aspects of society across the entire world. Globally as of July $5^{\text {th }}, 2021$, there have been 184000000 confirmed cases of COVID-19, which tragically include 3980000 deaths.

Sars-CoV-2 is spread through the respiratory system with the primary modes of transmission being aerosol, respiratory droplets, and direct contact.

It is of great importance to understand that one of the primary modes of transmission, the aerosol (bioaerosol) mode is a mode that is omnipresent in most dental settings.

To prevent dissemination of disease in American dental offices, the CDC and the ADA have developed strict protocols for dental care providers to follow.

Protocols include case selection, protective equipment (PPE), Covid testing, procedure risk stratification, and reduction of aerosols. Laser-assisted dentistry has been identified as a potential key modality in the reduction of bio aerosols.

Keywords: Bio aerosol, COVID-19, dentistry, laser-assisted dentistry.
Published Online: September 04, 2021

ISSN: $2684-4443$

DOI: 10.24018 /ejdent.2021.2.5.86

Natalia Elson *

Clinical Assistant Professor, NYU College of Dentistry, New York, USA.

(e-mail: natalia.elson@gmail.com)

Irene Brandes

Clinical Assistant Professor, NYU College of Dentistry, New York, USA.

(e-mail: icb1@nyu.edu)

Steven Resnick

Clinical Assistant Professor, NYU College of Dentistry, New York, USA.

(e-mail: sjr10@nyu.edu)

*Corresponding Author

\section{INTRODUCTION}

Sars-COVID-19 is a self-limiting infectious disease. Of those people infected, $1.2 \%$ are asymptomatic, $80.9 \%$ have mild symptoms, $13.8 \%$ have severe symptoms, while $4.7 \%$ require critical care. Tragically the virus is the primary causation of death in $2.3 \%$ of those it infects [1].

While the majority of those infected recovers without the use of advanced therapies, this novel Coronavirus can cause severe symptoms leading to cytokine storms in people with underlying conditions including cardiac issues, diabetes, and obesity.

As stated previously the probable routes of transmission include droplets (more than 5 microns), airborne aerosol (less than 5 microns), and direct contact of surfaces, all modes being very common in dental settings.

COVID-19 virus particle itself is 0.06-0.14 microns. Most dental procedures create a high volume of aerosol in a closed environment. The intimately close distance between the patient and provider during both diagnosis and therapies puts dentists and their staff at risk [2].

To date, there are no reports of COVID-19 transmission in dental settings. However, given the high transmissibility of disease, all dental personnel should take all precautions to protect themselves, their patients, and their family.

In response to the high transmissibility rate of the virus, every dental clinic should update their infection control protocol, alter their scheduling and treatment times, and consider the implementation of new equipment, modalities, and materials. The education of patients and employees regarding the COVID-19 routes of transmission lies within the dentist's purview and responsibility.

Coronaviruses enter human cells through an interaction with angiotensin converting enzyme 2 (ACE-2) [3].

The vast majority of ACE- 2 reside on the tongue and in the lungs. Virome is a normal inhabitant of the healthy oral microbiome residing in the gingival sulcus in symbiotic relationship with bacteria. Diverse population of DNA and RNA viruses are found in saliva and sub gingival plaque. However, the virome is more individual and gender specific than bacteria with remarkable colonization stability. Viral DNA and viable virus can be detected in saliva in early disease before pulmonary changes.[4], [5].

In most viral infections there is no viral shedding after resolution of the symptoms. Both Influenza A and Influenza $\mathrm{B}$ are exceptions.

According to the CDC recovered persons can continue to shed detectable Sars-CoV-2 RNA in upper respiratory specimens for up to 3 months after illness onset, albeit at concentrations considerably lower than during illness, in ranges where replication-competent virus has not been reliably recovered and infectiousness is unlikely. ACE-2 is a cellular doorway for COVID-19. The gingival sulcus is a niche and most ACE-2 in the oral cavity can be found in the tongue, oral mucosa, guns, salivary glands, taste buds and respiratory mucosa. 


\section{Changes in Clinical Protocol}

The coronavirus pandemic has brought tremendous changes in Dentistry due to the possibility of viral transmission through aerosol or contaminated surfaces. New infection control protocols have reinforced existing protective measures and have changed Dentistry both currently and perhaps going forward into the future [6].

Various dental procedures produce varying amounts of aerosols and droplets. Understanding these concepts allows us to classify risks of airborne contamination into low, moderate, and high-risk categories. And sonic scalers represent the greatest source of contamination while laser devices represent the lowest source [7].

Dentists have long worked diligently to protect their environment from viruses and bacterial transmissions, both bloodborne and respiratory. Many of these pre COVID preventive procedures are, unfortunately, inadequate to prevent the transmission of Sars-CoV-2 [8].

Dentists are providing care in a "wet" environment that includes saliva, water coolant, prover, and patient normal breath, coughing, sneezing, and gagging. Dental units water coolant produces spray containing splatter, droplets, dropletsnuclei, true aerosol, and a combination of the aforementioned items. During the high-risk aerosol procedures, bioaerosol is created and has the potential to spread up to two meters[9]. It returns to pre-operative levels within $30 \mathrm{~min}$ to 2 hours. Bio aerosol is a combination of blood, saliva, water, tooth or tissue structures, plaque, dental materials, and 38 different variant types of microorganisms. It is both tissue and caries level specific. The peak of exposure time is 15 minutes. The utilization of surgical level masks fitted N95 respirators, face shields, bouffant, shoe covers, and other personal protective equipment are mandatory enhancements over the pre-COVID protective model [10].

Some facts:

- Aerosolized water from most dental equipment is 0.001 to 50 microns in size.

- Microorganism level in the atmosphere surrounding patient care returns to normal between 10 to $30 \mathrm{~min}$. But 5 microns COVID-19 particles can remain viable for up to 3 hours.

- Half-life of the particle in aerosol is 1.1 hours.

- Sars-CoV RNA in saliva manufactures $7.08 \times 10^{3}$ to $6.38 \times 10^{8}$ copies/mL [11].

- Dental laser plume is 0.1 micrometer in size.

\section{AEROSOL REDUCTION}

When viewing all of the previously mentioned data in a global fashion, it seems both logical and expeditious to consider the reduction of the level of aerosol as a primary methodology of mitigating the risk of transmission. Dentists should be aware of all procedures that can reduce and diminish the possibility of contamination during aerosol generation [12].

The layering protective protocol would help to prevent disease spread.

- The first layer is a PPE;

- The second layer is a pre-procedural rinse;

- The third layer is-HVE, HEPA, ULPA, UVC, etc.
Natural air ventilation $-60 \mathrm{~L} / \mathrm{sec}$ per patient [13]. Each dental appointment should start with patient triage: first time over the phone night before the appointment and second time in the dental setting along with body temperature registration. Use of antimicrobial mouth rinse prior to dental treatment such as $0.5-1.5 \%$ hydrogen peroxide or $0.2 \%$ Povidone iodine can reduce viral load in saliva. All aerosol producing dental procedures should be performed under the rubber dam if possible. Thorough disinfection of all contaminated surfaces between the patients is highly recommended with $0.1 \%$ Sodium hypochlorite or $70 \%$ isopropyl alcohol. That is why at least a 15-20-minute time frame between the patients should be implemented in scheduling to achieve this goal [14].

It is also important to limit the number of patients in the reception area. After each patient at least $10 \mathrm{~min}$ for 20 square meter room air change is recommended. UVGI with short wavelength (180-280 $\mathrm{nm}$ ) shows a promising result in air disinfection.

HVE (extra and intra oral) with diameter at least $8 \mathrm{~mm}$ is a must to have device in each dental operation.

The device should be held at the proper distance $(6-15 \mathrm{~mm})$ from the active ultrasonic tip.

HEPA, ULPA, Carbon filter-all these air filtration devices can remove particles of 0.3 micrometer. The main disadvantages are maintenance and price. [15]

\section{A. Laser Assisted Dentistry and Aerosol}

Laser-assisted dentistry on the other hand can be beneficial in many ways in this uncertain period and in the future.

Among the other dental laser classifications, we can divide them to an aerosol-producing (Er,Cr:YSGG; Er:YAG; CO2$9.3 \mathrm{~nm}$ ) and non-aerosol producing (Nd:YAG, CO2-10.3 nm, diodes) [16]. Both these types have advantages and disadvantages.

The biggest disadvantages of the most high-power lasers are their cost and mandatory training. But laser implementation in the dental practice allows the practitioner to perform less painful procedures, reduce the amount of anesthesia, achieve better treatment outcomes, and reduce the amount of antibiotics and painkiller medications usage. Do high-power lasers produce aerosols? Yes. But all laser devices are producing the plume [17].

According to Academy of Laser Dentistry position paper: aerosol (plume) is a by-product due to laser-tissue interaction. It contains organic and inorganic matters, viruses, toxic gases, and chemicals. Nominal hazard zone is unique for each laser type.

Dentists should be aware of safety rules and implement the laser safety protocol and assign safety officers.

The rubber dam usage is a must. Special attention to rubber dam color and clamp material is necessary. Dark rubber dam absorbs the energy produced by laser and metal clamps create the reflection. HVAC and N95 mask or N99 respirator (preferable) and face shield have always been used in laserassisted dentistry but now became a standard when any dental procedures are performed [18].

A few studies have been done about possible disease transmission by laser plume, including transmission of HPV virus. But the advantages of using lasers in dentistry override the potential harm especially if all precautions are taken. 
Laser bacterial reduction by directly affecting biofilm can be used as a pre-procedural tool to reduce the bacterial and viral levels in the period pockets and potentially in the producing aerosol.

Photo biomodulation and photodynamic therapy in the COVID-19 era are promising addition to dental treatment. Lasers have been shown to have stimulatory effects on cells.

According to Biolase Inc. research, lasers produce $98 \%$ less aerosol compared to traditional high-speed handpieces utilizing the ISO 14457 recommended flow rate. Biolase also claimed that lasers produce $80 \%$ less water than traditional handpieces. With the proper training dentists and dental hygienists could successfully implement laser protocol in their everyday treatment and benefit their patients and protect the environment [19].

\section{B. Possible Novel Approach in New Normal}

From a dental industry perspective, equipment, and product manufacturers and suppliers are building upon and further developing, the industry's extremely close relationship and partnership with the dental profession, in order to develop and implement new ways of thinking and new approaches, products, and techniques to protect patients and clinicians, focusing on a return to the more widespread provision of dentistry.

There are multiple ways lasers can be incorporated in the dental practice but in COVID-19 era when treatment of this disease is still uncertain photo biomodulation showed promising results in fighting against Cytokine storm. Local effects on cells are stimulation of proliferation, ATP induction, DNA and RNA synthesis, NO release, activation of cell signaling cascades), c oxidase activation, calcium flux, stress protein expression, and modification of intracellular organelle. The general effect is metabolic, analgesic, antiinflammatory, immunomodulatory [20].

Blue, ultraviolet, violet wavelengths can decrease microorganism directly.

aPDT - Antimicrobial Photodynamic Therapy is a method which combines photosensitizers and laser applications. Among others the most useful medications in laser assisted Dentistry are curcumin, methylene blue, vitamin B2, and indocyanine. The mechanism of action: lack of long-term toxicity, gene mutation, ability to remove microorganisms in a very short period of time, less damage to adjacent tissues, access to areas with complex anatomy, low risk of bacteremia, high repeatability without bacterial and viral resistance.

\section{DISCUSSION}

Sars-COVID-19 puts the whole world in a new reality now and forever. We as a dentist have always been aware of harmful potentials of aerosol aka bioaerosol we are producing during the treatment, and we were following the Universal precaution infection control protocol [21]. Awareness of society about safety during dental treatment gives us an opportunity to implement new methods and techniques. On the other hand, to protect ourselves, our staff, and community. Everything starts with education. Dental schools might need to update the curriculum by implementing not just anti COVID-19 infection control protocol and new treatment modalities but make the new approach in Dental education as it.

\section{REFERENCE}

[1] Wei-Kung Wang, Detection of SARS-associated Coronavirus in Throat Wash and Saliva in Early Diagnosis, Emerg Infect Dis., 2004, Jul; 10(7): 1213-1219. doi: 10.3201/eid1007.031113.

[2] S. Tang, Y. Mao, R. M. Jones, Q. Tan, J. S. Ji, N. Li, J. Shen, Y. Lv, L. Pan, P. Ding, X. Wang, Y. Wang, C. R. MacIntyre, X. Shi, "Aerosol transmission of SARS-CoV-2? Evidence, prevention, and control," Environ Int, 144:106039, 2020.

[3] Kaycee M. Sink, Xiaoyan Leng, Jeff Williamson, Stephen B. Kritchevsky, Kristine Yaffe, Lewis Kuller, Sevil Yasar, Hal Atkinson, Mike Robbins, Bruce Psaty, David C. Goff Jr, Angiotensin-Converting Enzyme Inhibitors and Cognitive Decline in Older Adults With Hypertension: Results From the Cardiovascular Health Study, AMA Internal Medicine Original Investigation, July 13, 2009.

[4] P. S. Kumar, K. Subramanian, "Demystifying the mist: Sources of microbial bioload in dental aerosols," Journal of periodontology, 91(9), 1113-1122, 2020.

[5] S. Tang, Y. Mao, R. M. Jones, Q. Tan, J. S. Ji, N. Li, and X. Shi, "Aerosol transmission of SARS-CoV-2? Evidence, prevention, and control," Environment international, 144, 106039, 2020.

[6] A. Amato, M. Caggiano, M. Amato, G. Moccia, M. Capunzo, and F. De Caro, "Infection control in dental practice during the COVID-19 pandemic," International journal of environmental research and public health, 17(13), 4769, 2020

[7] J. B. Franco, P. F. Ribas, L. A. S. V. Júnior, D. T. Matias, B. L. R Varotto, C. R. Hamza, ... and M. P. S. de Melo Peres, "Hospital Dentistry and Dental Care for Patients with Special Needs: Dental approach during COVID-19 Pandemic," Brazilian Dental Science, 23(2), 9-p, 2020.

[8] C. C. Chen, and K. Willeke, "Aerosol penetration through surgical masks," American journal of infection control, 20(4), 177-184, 1992.

[9] S. Tang, Y. Mao, R. M. Jones, Q. Tan, J. S. Ji, N. Li, and X. Shi, "Aerosol transmission of SARS-CoV-2? Evidence, prevention and control," Environment international, 144, 106039, 2020.

[10] A. Giudice, F. Bennardo, A. Antonelli, S. Barone, L. Fortunato, "COVID-19 is a new challenge for dental practitioners: advice on patients' management from prevention of cross infections to telemedicine," The Open Dentistry Journal, 14(1), 2020.

[11] R. W. K. Li, K. W. C. Leung, F. C. S. Sun, and L. P. Samaranayake, "Severe Acute Respiratory Syndrome (SARS) and the GDP. Part I Epidemiology, virology, pathology and general health issues," British dental journal, 197(2), 77-80, 2004.

[12] A. Liebert, B. Bicknell, W. Markman, and H. Kiat, "A potential role for photobiomodulation therapy in disease treatment and prevention in the era of COVID-19," Aging and disease, 11(6), 1352, 2020.

[13] A. A. Lushnikov, and A. E. Negin, "Aerosols in strong laser beams," Journal of aerosol science, 24(6), 707-735, 1993.

[14] R. W. K. Li, K. W. C. Leung, F. C. S. Sun, and L. P. Samaranayake, "Severe acute respiratory syndrome (SARS) and the GDP. Part II: implications for GDPs," British dental journal, 197(3), 130-134, 2004

[15] P. S. Hughes, "Boiling and splattering liquid with the Er: YAG laser," Lasers in surgery and medicine, 23(5), 248-249, 1998.

[16] M. V. L. Ferreira, and L. N. de Souza, "High power laser use in the COVID-19 pandemic era in dentistry: tips for the readers," Lasers in Dental Science, 4(3), 165-166, 2020.

[17] B. L. Ziegler, C. A. Thomas, T. Meier, R. Müller, T. M. Fliedner, and L. Weber, L, "Generation of infectious retrovirus aerosol through medical laser irradiation," Lasers in Surgery and Medicine: The Official Journal of the American Society for Laser Medicine and Surgery, 22(1), 37-41, 1998.

[18] S. Erzinger, K. G. Schueepp, J. Brooks-Wildhaber, S. G. Devadason, and J. H. Wildhaber, "Facemasks and aerosol delivery in vivo," Journal of Aerosol Medicine, 20(s1), S78-S84, 2007.

[19] https://www.biolase.com/lessismore/ Less is More.

[20] R. Fekrazad, "Photobiomodulation and antiviral photodynamic therapy as a possible novel approach in COVID-19 management,", 2020.

[21] J. M. Garden, M. K. O'Banion, A. D. Bakus, and C. Olson, "Viral disease transmitted by laser-generated plume (aerosol)," Archives of dermatology, 138(10), 1303-1307, 20. 